\title{
Genetics Bases of Cardiac Sodium Channel Mutations linked to Inherited Cardiac Arrhythmias
}

\author{
Hector Barajas-Martinez ${ }^{1}$, Dan Hu${ }^{1}$, Adrian Baranchuk ${ }^{2}$
}

DOI: http://dx.doi.org/10.7322/jhgd.123189

To the Editor:

This issue of J Hum Growth Dev contains an excellent review by Pérez-Riera et $\mathrm{al}^{1}$ describing one of the most involved ion channel gene named SCN5A that encoded the cardiac sodium channel linked to different inherited cardiac arrhythmias (ICA) phenotypes or/and syndromes. The genetic bases of mutation in the SCN5A gene has been implicated in ICA phenotypes and their functional impact may lead to a significant loss (reduction) or gain (increasing) of the sodium channels mutants compared with the wild type.

Sodium channelopathies are recently delineated, emerging as a group of ICA disorders grouped by genetically determined defects in ion-channel function. ICA disorders are characterized by a prominent genetic and phenotypic heterogeneity that can make them challenging to understand. This systematic review by Pérez-Riera et al (1) attempts to understand the role of the sodium channel mutations of these disorders according to their clinical manifestations (i.e., Long QT Syndrome, Brugada Syndrome and others), within the context of what is presently known and their molecular and genetic basis. The review is intended to assist clinicians and scientists in enhancing their clinical and genetic diagnostic knowledge.

The functional genomics in vitro studies are part of the new process to be translated into potential treatments to restore the dysfunction cardiac electrophysiology linked within mutations in the SCN5A gene ${ }^{2}$. The new generation of DNA sequencing methods has now discovered hundreds of new mutations in different sodium channels genes that can lead different phenotypes of channelopathies; and the majority are located in the SCN5A gene showing low expression in the adult heart system. Our group and others found new mutations in the $\beta 3$ subunits associated with Brugada syndrome and cardiac conduction disease with a significant reduction in the peak sodium current by the co-interaction with the mutant $\beta$ subunits and SCN5A channels ${ }^{3}$.

Pérez-Riera et al; described in this review that differentmutations in SCN5Amayleadalarge spectrum of different arrhythmia phenotypes ${ }^{1}$. For this reason, it is very important to develop new innovative studies in pharmacogenomics to determine the best treatment options to be

\footnotetext{
1 Molecular Genetics Department, Masonic Medical Research Laboratory, Utica, New York, USA.

2 Heart Rhythm Service, Queen University, Kingston, Ontario, Canada.

Corresponding author: Hector Barajas-Martinez - Email: barajash@mmrl.edu
}

Suggested citation: Barajas-Martinez H, Hu D, Baranchuk A. Genetics Bases of Cardiac Sodium Channel Mutations linked to Inherited Cardiac Arrhythmias Genetics. J Hum Growth Dev. 26(2): 277-280. J Hum Growth Dev. 26(3): 277-280. Doi: http://dx.doi.org/10.7322/ jhgd.123189

Manuscript submitted: Oct 16 2016, accepted for publication Nov 142016. 
used as specific antiarrhythmic drugs (AAD) in all different ICA phenotypes or syndromes. The majority of the AAD agents that act as blockers have receptor sites on the alpha subunits in the sodium channel protein structure. (4) There are some ADD that shown a positive result by rescuing the loss of function in some SCN5A mutations. For example, the I1660V mutation that produced a significant reduction in sodium current can be treated by using some sodium channel blockers that can rescue the $11660 \mathrm{~V}$ mutant sodium current (mexiletine, ranolazine, ajmaline, quinidine). Mexiletine has proved to be the most effective. ${ }^{5}$ In the modeling site of sodium channels; the L325R mutation showed in cardiac action potential experiments in patients with reduced sodium channels and fever; conditions that could prematurely shorten the action potential, suggesting that a dominant negative phenomenon may underlie $\mathrm{BrS}$ triggered by fever. (6) On the other hand, some other AAD are used to unmask other diseases such as BrS.

We are very grateful to read the integration of the basic and translational description of this review by Perez-Riera et al; which contributes to knowledge translation in this fascinating are of science. 
1. Pérez-Riera AR, Raimundo RD, Watanabe RA, Figueiredo JL, Abreu LC. The cardiac sodium channel its mutations and their spectrum arrhythmia phenotypes. J Hum Growth Dev. 2016; 26(3): 281-96. DOI: http://dx.doi.org/10.7322/jhgd.122759

2. Barajas-Martínez HM, Hu D, Cordeiro JM, Wu Y, Kovacs RJ, Meltser H, et al. Lidocaineinduced Brugada syndrome phenotype linked to a novel double mutation in the cardiac sodium channel. Circ Res. 2008;103(4):396-404. DOI: http://dx.doi.org/10.1161/ CIRCRESAHA.108.172619

3. Hu D, Barajas-Martinez H, Burashnikov E, Springer M, Wu Y, Varro A, et al. A mutation in the $\beta 3$ subunit of the cardiac sodium channel associated with Brugada ECG phenotype. Circ Cardiovasc Genet. 2009;2(3):270-8. DOI: http://dx.doi.org/10.1161/ CIRCGENETICS.108.829192

4. Cordeiro JM, Barajas-Martinez H, Hong K, Burashnikov E, Pfeiffer R, Orsino AM, et al. Compound heterozygous mutations P336L and I1660V in the human cardiac sodium channel associated with the Brugada syndrome. Circulation. 2006; 114(19): 2026-33. DOI: http://dx.doi.org/10.1161/CIRCULATIONAHA.106.627489

5. Keller DI, Rougier JS, Kucera JP, Benammar N, Fressart V, Guicheney P, et al. Brugada syndrome and fever: genetic and molecular characterization of patients carrying SCN5A mutations. Cardiovasc Res. 2005;67(3):510-9. DOI: http://dx.doi.org/10.1016/j. cardiores.2005.03.024

6. Moreau A, Keller DI., Huang H, Fressart V, Schmied C, Timour Q, et al. Mexiletin differentially restores the trafficking defects caused by two Brugada syndrome mutations Front Pharmacol. 2012;3:62. DOI: http://dx.doi.org/10.3389/fphar.2012.00062 